PPPL-3468

PPPL-3468

UC-70

Theory, Simulation, and Experimental Studies of Zonal Flows

by

T.S. Hahm, K.H. Burrell, Z. Lin, R. Nazikian, and E.J. Synakowski

July 2000

$\int D \sqrt{D} \int \sqrt{\zeta} \int\left[\begin{array}{l}\text { PAINCETON } \\ \text { PLABMA PHYSICS } \\ \text { LABOAATOAY }\end{array}\right.$

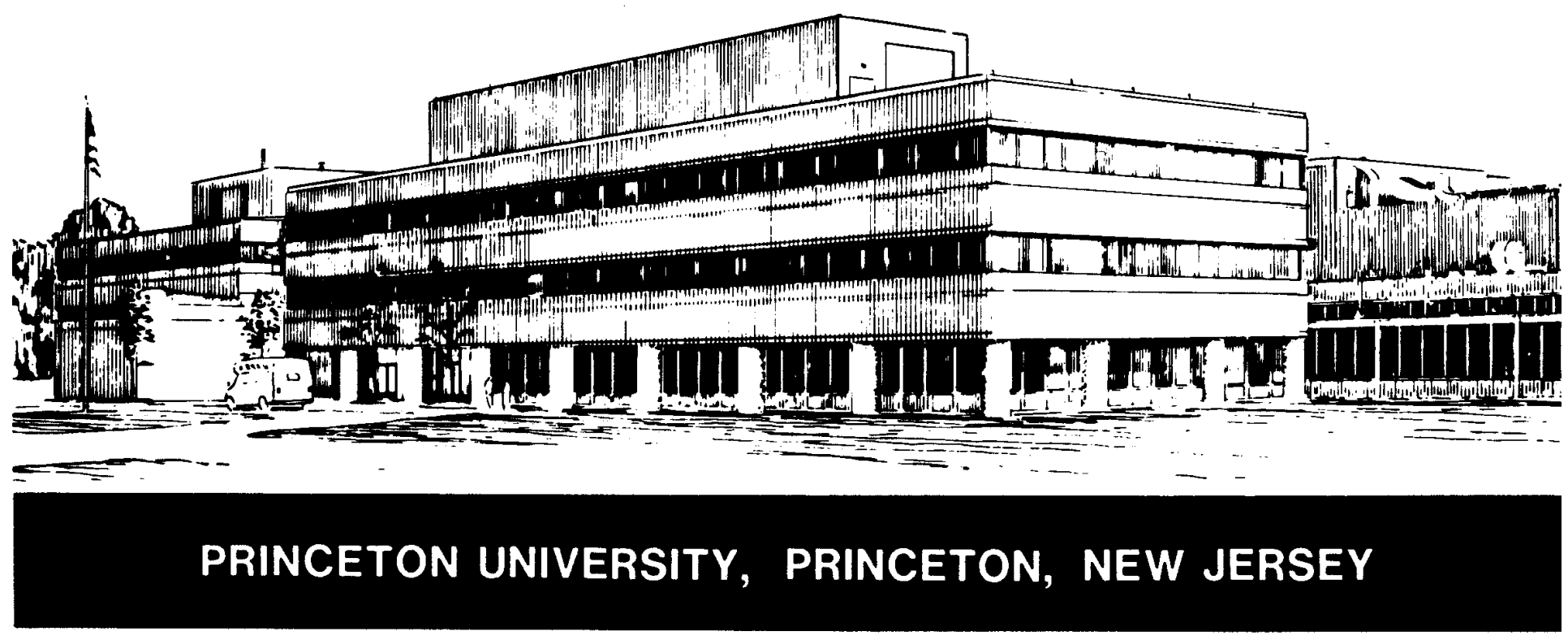




\section{PPPL Reports Disclaimer}

This report was prepared as an account of work sponsored by an agency of the United States Government. Neither the United States Government nor any agency thereof, nor any of their employees, makes any warranty, express or implied, or assumes any legal liability or responsibility for the accuracy, completeness, or usefulness of any information, apparatus, product, or process disclosed, or represents that its use would not infringe privately owned rights. Reference herein to any specific commercial product, process, or service by trade name, trademark, manufacturer, or otherwise, does not necessarily constitute or imply its endorsement, recommendation, or favoring by the United States Government or any agency thereof. The views and opinions of authors expressed herein do not necessarily state or reflect those of the United States Government or any agency thereof.

\section{Availability}

This report is posted on the U.S. Department of Energy's Princeton Plasma Physics Laboratory Publications and Reports web site in Calendar Year 2000. The home page for PPPL Reports and Publications is: http://www.pppl.gov/pub_report/

DOE and DOE Contractors can obtain copies of this report from:

U.S. Department of Energy

Office of Scientific and Technical Information

DOE Technical Information Services (DTIS)

P.O. Box 62

Oak Ridge, TN 37831

Telephone: (865) 576-8401

Fax: (865) 576-5728

Email: reports@adonis.osti.gov

This report is available to the general public from:

National Technical Information Service

U.S. Department of Commerce

5285 Port Royal Road

Springfield, VA 22161

Telephone: $1-800-553-6847$ or

(703) $605-6000$

Fax: (703) 321-8547

Internet: http://www.ntis.gov/ordering.htm 


\title{
Theory, Simulation, and Experimental Studies of Zonal Flows
}

\author{
T. S. Hahm ${ }^{1}$, K. H. Burrell ${ }^{2}$, Z. Lin ${ }^{1}$, R. Nazikian ${ }^{1}$, and E.J. Synakowski ${ }^{1}$ \\ ${ }^{1}$ Princeton University Plasma Physics Laboratory, Princeton, NJ 08543, USA \\ ${ }^{2}$ General Atomics, San Diego, CA92186-9784, USA
}

We report on current theoretical understanding of the characteristics of self-generated zonal flows as observed in nonlinear gyrokinetic simulations of toroidal ITG turbulence [Science 281, 1835 (1998)], and discuss various possibilities for experimental measurements of signature of zonal flows.

\section{Physics of Random Shearing by Zonal Flows}

During the past several years, a lot of progress has been made in understanding the role of self-generated zonal flows in nonlinearly regulating Ion Temperature Gradient (ITG) turbulence, and associated transport. The self-generated zonal flows are radially localized $\left(k_{r} a>>1\right)$, axisymmetric $\left(k_{\phi}=0\right)$, and mainly poloidal $\mathbf{E} \times \mathbf{B}$ flows, $\mathbf{u}_{E}$. Their high $k_{r}$ components can vary in the eddy turnover time scale[1] unlike externally driven macroscopic $\mathbf{E} \times \mathbf{B}$ flows which vary in much slower time scale.

An analysis of the nonlinear gyrofluid simulation results has indicated that the instantaneous $\mathbf{E} \times \mathbf{B}$ shearing rate associated with self-generated zonal flows exceed the maximum linear growth rate by an order of magnitude, while the turbulence fluctuation amplitude remained definitely above thermal noise level and the ion thermal transport remained anomalous. This was somewhat puzzling since in the case with the macroscopic $\mathbf{E} \times \mathbf{B}$ shear flows which are now routinely measured or calculated in existing toroidal devices, their plasmas made transition to enhanced confinement regimes[2] when the $\mathbf{E} \times \mathbf{B}$ shearing rate in general toroidal geometry[3] $\omega_{E}^{(0)}=\frac{\left(R B_{\theta}\right)^{2}}{B} \frac{\partial}{\partial \psi}\left(\frac{E_{r}^{(0)}}{R B_{\theta}}\right)$ exceeds the linear growth rate of microinstabilities in the absence of the $\mathbf{E} \times \mathbf{B}$ shear.

To understand this qualitative difference between the nonlinear simulation results with fluctuating zonal flows and the experimental results regarding the equilibrium $\mathbf{E} \times \mathbf{B}$ flows, we have considered a model problem[4] in which we assumed that the potential for zonal flow varies sinusoidally in time with a characteristic frequency $\omega_{f}$. We have shown that fast time varying components of zonal flows are less effective in shearing turbulence eddies. The fundamental reason for this is that the zonal flow shear pattern changes before the eddies can be completely torn apart. The turbulent eddies can then recover some of their original shape, and the shearing effect is reduced. An effective shearing rate including this effect has been derived analytically and evaluated from the long term gyrofluid simulations[4]. Overall, it was somewhat below but comparable to the linear growth rate[4], qualitatively consistent with considerable reduction[5], not total suppression of turbulence observed in simulations. It is worth noting that a similar behavior has been observed experimentally just prior to H-mode transition[6]. Our gyrokinetic simulations show significant broadening of $k_{r}$ spectrum due to zonal flows as indicated in Fig. 1. 


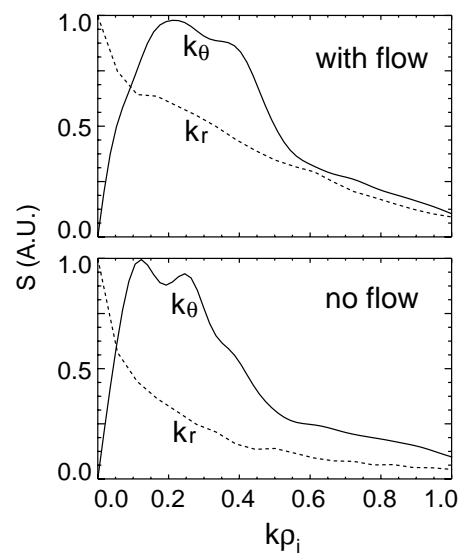

Figure 1: $k_{r}$ spectrum of ambient turbulence from gyrokinetic simulation is broadened due to random shearing of eddies by self-generated $\mathbf{E} \times \mathbf{B}$ flows.

These are in qualitative agreements with our analytical prediction that the radial correlation length $\Delta r \equiv \Delta \psi / R B_{\theta}$, is reduced by the flow shear relative to its value $\Delta r_{0} \equiv \Delta \psi_{0} / R B_{\theta}$, determined by ambient turbulence alone:

$$
\left(\frac{\Delta \psi_{0}}{\Delta \psi}\right)^{2}=1+\frac{\omega_{E f f}^{2}}{\Delta \omega_{T}^{2}}
$$

Therefore, we expect that considerable fluctuation reduction occurs when the following effective shearing rate $\omega_{E f f}$ becomes comparable to the decorrelation rate of the ambient turbulence, $\Delta \omega_{T}$.

$$
\omega_{E f f} \equiv \omega_{E}^{(0)} \frac{\left((1+3 F)^{2}+4 F^{3}\right)^{1 / 4}}{(1+F) \sqrt{(1+4 F)}}
$$

Here $\omega_{E}^{(0)}$ is the instantaneous $\mathbf{E} \times \mathbf{B}$ shearing rate. $R \Delta \phi$ is the correlation length in toroidal direction, and $F \equiv \omega_{f}^{2} / \Delta \omega_{T}^{2}$. When $E_{r}$ varies slowly enough such that $F<<1$, we have $\omega_{E f f}=\omega_{E}^{(0)}$, and recover the previous result in general toroidal geometry[3]. On the other hand, when $E_{r}$ varies fastly in time such that $F>>1$, we have $\omega_{E f f}<<\omega_{E}^{(0)}$. In this case, it is difficult to achieve turbulence reduction. This summarizes our theoretical understanding of random shearing process at a kinematic level. Other characteristics of zonal flows observed in gyrokinetic simulations are summarized in Ref.[7].

A statistical theory based on drift wave kinetics in random media consisting of an ensemble zonal flows[8] also predicts broadening of $k_{r}$ spectrum by random refraction. In that work, a turbulence plasma is considered as a self-regulating two component system consisting of the usual ambient ITG turbulence and $\left(k_{\phi}, k_{\theta}\right)=(0,0)$ component which is zonal flow. Since zonal flow cannot tap the expansion free energy associated with pressure gradient directly, the energy lost by turbulence by random shearing is gained by zonal flows as illustrated in Fig 2. Although we usually discuss each process independently, duality of random shearing and flow generation is just a statement on the nonlinear mode coupling, specifically a distant interaction between finite $k_{\theta}$ and zero $k_{\theta}$ modes[8]. Therefore, a bi-spectral analysis of fluctuation data could be useful in demonstrating the existence of zonal flows[9]. 


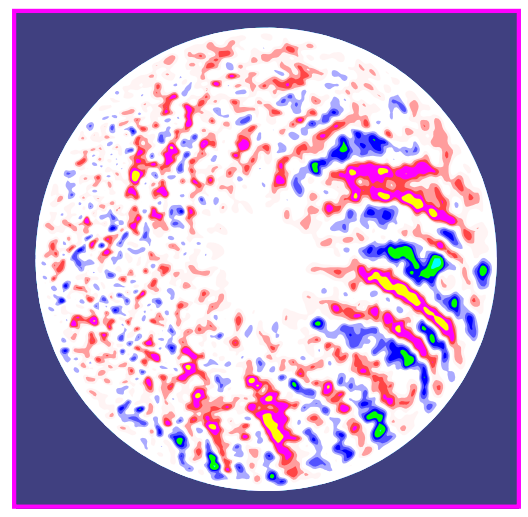

Random Shearing
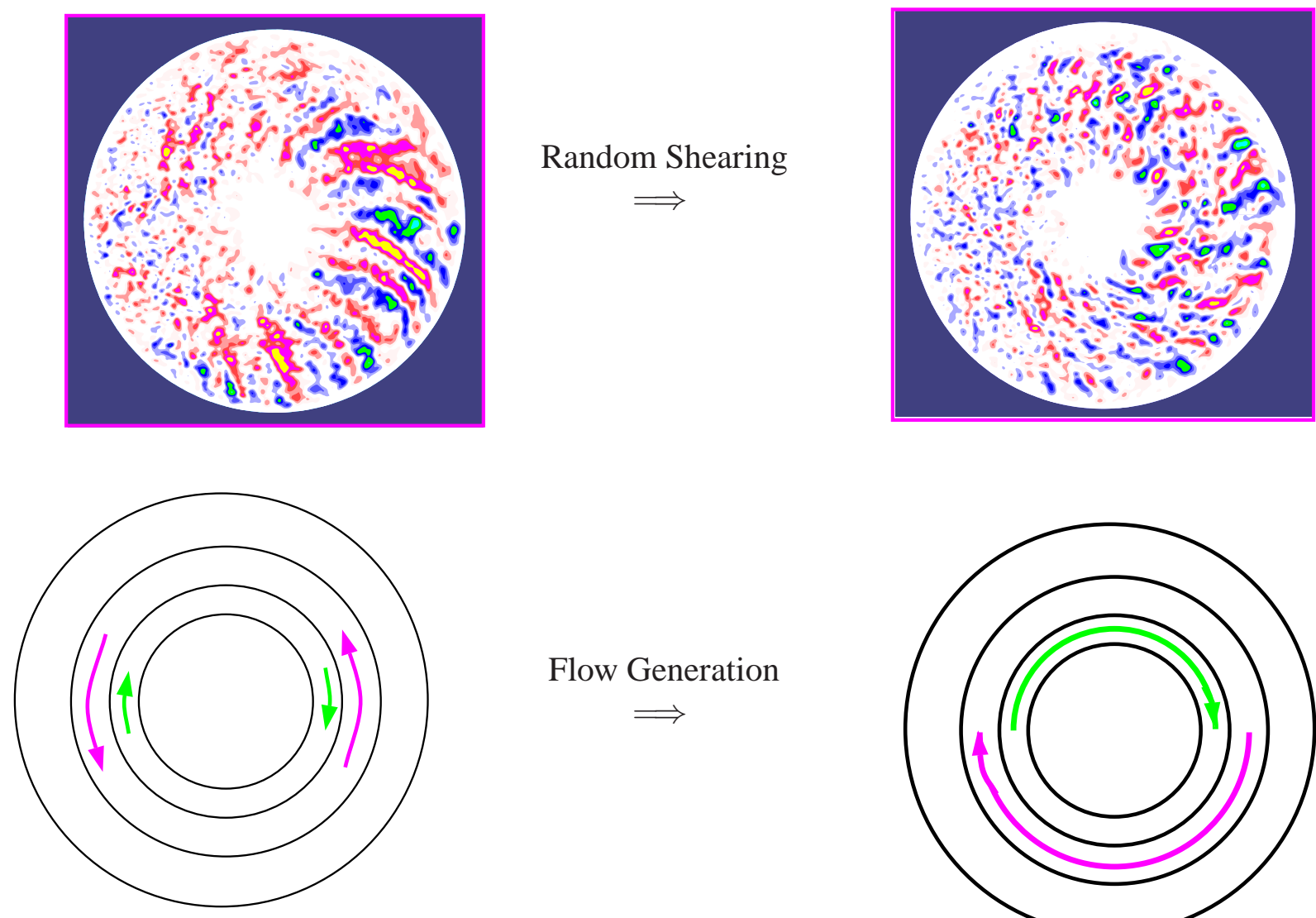

Flow Generation

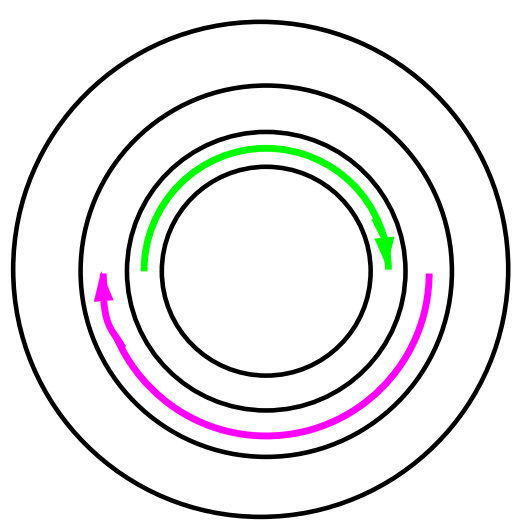

Fig. 2. Self-regulating ITG turbulence-zonal flow system

\section{Search for Signature of Zonal Flows}

Since it requires an order of magnitude improvement in temporal resolution to measure the zonal flows with an autocorrelation rate of the order of $5 \mathrm{KHz}$ directly in the core plasma using the present Spectroscopy or Motional Stark Effect diagnostics capabilities, it is useful to look

for signatures of zonal flows on the ambient turbulence[7]. One way to systematically demonstrate the effects of zonal flows is to change plasma parameters in such a way as to change the zonal flow intensity while keeping the linear instability properties

nondimensionally similar, and show the agreement between experiments (from Beam Emission Spectroscopy, reflectometry, microwave scattering, and 3-d microwave imaging) and theory (from gyrokinetic simulations with collisions) of ambient turbulence spectra of $k_{r}$ and $k_{\theta}$. Systematic nondimensional $\nu_{*}$ scan experiments will be illuminating, since a realistic level of ion-ion collisions in the core plasma will affect only the zonal flow damping, not the ITG or Trapped Ion Mode linear growth rate[10]. As the ion-ion collsions are increased, with other parameters fixed in gyrokinetic simulations, an decrease in the ambient turbulence spectra of $k_{r}$ and an increase in ion thermal transport have been observed. The increase in ion thermal transport is in qualitative agreement with trends from previous $\nu_{*}$ scan of DIII-D H-mode plasmas[11] and C-Mod H-mode plasmas[12]. It is remarkable that bursty behavior of density fluctuations with a period close to collisional damping time of flows has been also observed in simulations[13]. The observed bursting period $(\sim 3 m s)$ in TFTR Reversed Shear plasmas[13]

is also close to the collisional damping time of zonal flows as shown in Fig. 3. Bursting behavior in the experiments could be used for zonal flows studies since it naturally exhibits 
both the high zonal flow-low ambient turbulence state and the low zonal flow-high ambient turbulence state, and thus facilitates the scalings studies in simulations without a need for changing the equilibrium profiles.

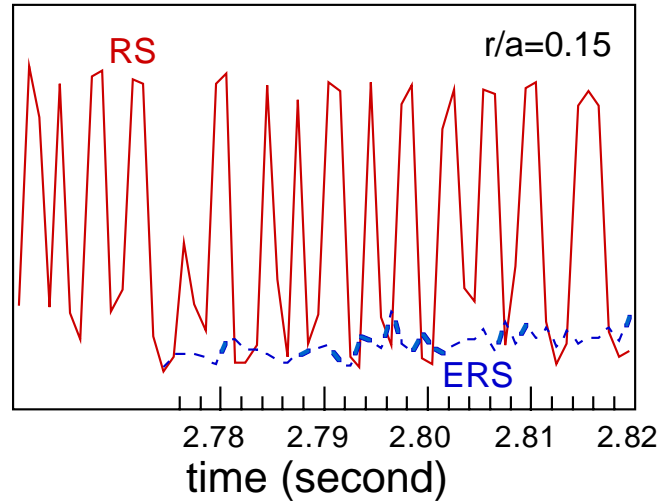

[Mazzucato, et al., PRL, 1996]

large bursts of fluctuation in TFTR RS plasmas observed period $\sim$ collisional flow damping time

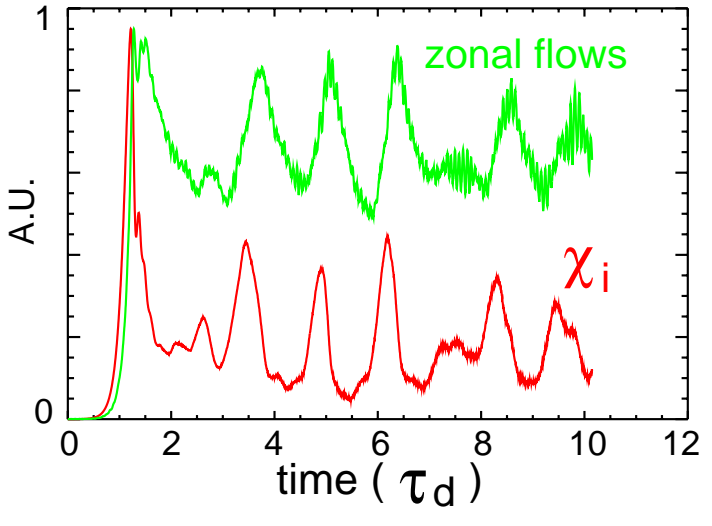

[Lin, et al., PRL, 1999]

collisional damping of zonal flows causes bursts of turbulent transport in gyrokinetic simulations

Fig. 2. Bursting of fluctuations have been observed in both experiments and simulations

\section{Acknowledgments}

The authors would like to thank M. Beer, P.H. Diamond, and E. Mazzucato for useful discussions. This work was supported by the U.S. Department of Energy Contract No. DE-AC02-76-CHO-3073 and the U.S. Department of Energy Contract No.

DE-AC03-89ER51114.

[1] M.A. Beer, Ph.D. Thesis, Princeton University, 1995.

[2] K.H. Burrell, Phys. Plasmas, 4, 1499 (1997).

[3] T.S. Hahm and K.H. Burrell, Phys. Plasmas 2, 1648 (1995).

[4] T.S. Hahm, M.A. Beer, Z. Lin, et al., Phys. Plasmas 6, 922 (1999).

[5] Z. Lin, T.S. Hahm, W.W. Lee,et al., Science 281, 1835 (1998).

[6] M.G. Shats, Plasma Phys. Controlled Fusion 41, 1357 (1999).

[7] T.S. Hahm, K.H. Burrell, Z. Lin,et al.,Plasma Phys. Controlled Fusion 42, A205 (2000). [8] P.H. Diamond et al., in Plasma Phys. and Controlled Nuclear Fusion Research

(IAEA, Vienna, 1998)IAEA-CN-69/TH3/1.

[9] P.H. Diamond, M.N. Rosenbluth, E. Sanchez,et al., Phys. Rev. Lett. 84, 4842 (2000).

[10] Z. Lin, T.S. Hahm, W.W. Lee, et al., Phys. Rev. Lett. 83, 3645 (1999).

[11] C. Petty and T. Luce, Phys. Plasmas 6, 909 (1999).

[12] M. Greenwald et al., Plasma Phys. Controlled Fusion 40, 789 (1998).

[13] E. Mazzucato et al., Phys. Rev. Lett. 77, 3145 (1996). 
The Princeton Plasma Physics Laboratory is operated by Princeton University under contract with the U.S. Department of Energy.

\author{
Information Services \\ Princeton Plasma Physics Laboratory \\ P.O. Box 451 \\ Princeton, NJ 08543
}

Phone: 609-243-2750

Fax: 609-243-2751

e-mail: pppl_info@pppl.gov

Internet Address: http://www.pppl.gov 